\section{Blasenkrebs vom Frühstücksei?}

Dass der Verzehr von gekochten Eiern in keinem Zusammenhang mit einem erhöhten Blasenkarzinomrisiko steht, zeigen chinesische Urologen. Sie analysierten 13 Studien mit insgesamt 184.727 Probanden. Die Vorliebe für Eier stand in keinem Zusammenhang mit mehr Blasenkrebserkrankungen. Allerdings wirkten sich gebratene Eier ungünstig aufs Krebsrisiko aus. Die Erklärung: Beim Braten entstehen karzinogene heterozyklische Amine. Außerdem steige mit dem Bratfett die Cholesterinmenge.

Li Fet al, Nutr Cancer 2013, 65:538-46

\section{Dicken Kindern brummt oft der Kopf}

Übergewicht ist bei Kindern auch mit Kopfschmerz assoziiert, berichten israelische Forscher: Eine Migräne wird deutlich häufiger bei übergewichtsgefährdeten $(60,4 \%)$ und übergewichtigen $(62,4 \%)$ Kindern festgestellt als bei Normalgewichtigen (34\%). Auch bei Spannungskopfschmerzen wurde deutlich: Mit steigendem BMI sind Kinder häufiger und stärker durch Schmerzattacken beeinträchtigt.

Ravid S et al, Headache 2013 (online first)

\section{Vitamin-D-Mangel im Sommer}

Vielen Schwangeren mangelt es auch im Sommer am Sonnenvitamin, wie eine Untersuchung mit 261 Schwangeren und 381 Neugeborenen zeigt. Die Konzentration von $50 \mathrm{nmol} /$ I Vitamin D im Blut bzw. Nabelschnurblut erreichten 49\% der Mütter und 39\% der Neugeborenen auch im Sommer noch nicht. Eine unzureichende Vitamin-D-Versorgung in der Schwangerschaft steht im Verdacht, Schwangerschaftskomplikationen zu begünstigen.

Wuertz C et al, British Journal of Nutrition (online first)

Sogar als Spray oder Rektalschaum

\title{
Kortison erhöht Thromboserisiko
}

Vor allem die systemische Applikation von Glukokortikoiden ist mit einem erhöhten Risiko für venöse Thromboembolien (VTE) assoziiert. Ein solcher, wenn auch weniger starker Zusammenhang wurde jetzt auch bei oraler Applikation und Verwendung von intestinal wirksamem Kortison beobachtet.

Um den Zusammenhang zwischen Glukokortikoidanwendung und VTE aufzudecken, werteten dänische Endokrinologen das nationale Patientenregister aus. Für die Studie standen Daten von fast 38.800 VTE-Patienten und mehr als 387.000 Kontrollpersonen zur Verfügung. 57,7\% der Patienten hatten eine idiopathische VTE. Bei mehr als $60 \%$ lag eine Beinvenenthrombose vor, fast $40 \%$ hatten eine Lungenembolie. Bei Patienten, die max. 90 Tage vor dem thrombotischen Ereignis mit systemischen Glukokortikoiden behandelt worden waren, war die Thromboseinzidenz um mehr als das Doppelte erhöht. Der Wert lag sogar noch höher (IRR 3,03), wenn nur jene Patienten betrachtet wurden, die erstmals mit Glukokortikoiden max. 90 Tage vor dem Ereignis behandelt worden waren. Lag die Behandlung in einem Zeitraum zwischen 91 Tagen und einem Jahr vor der VTE, errechnete sich die IRR zu einem Wert von 1,18 . Auch die inhalative Applikation (IRR 2,21) und die Anwendung intestinalwirksamer Glukokortikoide (IRR 2,17) waren mit einer erhöhten VTE-Inzidenz assoziiert.

(ple)

Johannesdottir SA et al, JAMA 2013, 173(9): 743-752

\section{Entlarvende Schlafstörung}

\section{Treten sagt Parkinson} voraus

Patienten mit einer REM-Schlafstörung erkranken offenbar alle früher oder später an Parkinson oder einer Lewy-Körperchen-Demenz. Darauf deuten Langzeitbeobachtungen hin.

Bei REM-Schlaf-Verhaltensstörungen (RBD, rapid-eye-movement sleep behaviour disorder) werden die Träume ausgelebt: die übliche motorische Hemmung in der REM-Phase unterbleibt, die Betroffenen schlagen und treten um sich. Was zunächst als lästige Parasomnie daherkommt, ist in Wirklichkeit aber der Beginn einer neurodegenerativen Erkrankung. Spanische Neurologen haben Daten von 44 neurologisch zunächst unauffälligen Patienten neu ausgewertet. Bei allen war zwischen 1991 und 2003 eine RBD diagnostiziert worden. Diese Patienten hatten die Ärzte bereits im Jahr 2005 untersucht und damals bei etwa 50\% Defizite gefunden. Sieben Jahre später hatte sich der Zustand der übrigen Patienten ebenfalls dramatisch verändert: Sieben von ihnen waren an Parkinson erkrankt, fünf an einer Lewy-Körperchen-Demenz und vier hatten kognitive Defizite. Nur bei vier der Überlebenden ließen sich noch keine klinischen Symptome feststellen, aber diese zeigten bereits Auffälligkeiten bei krankheitstypischen Biomarkern. Waren fünf Jahre nach der RBD-Diagnose noch 65\% der Patienten ohne neurologische Defizite, sank der Anteil nach zehn Jahren auf $27 \%$ nach 14 Jahren auf 7,5\%.

(mut)

Iranzo A et al, Lancet Neurol 2013, 12:443-53 